\title{
How to Design a 2D Active Grid for Dynamic Inflow Modulation
}

\author{
Tom T. B. Wester ${ }^{1}$ (D) . Johannes Krauss ${ }^{1}$ - Lars Neuhaus ${ }^{1} \cdot$ Agnieszka Hölling $^{1}$ • \\ Gerd Gülker $^{1} \cdot$ Michael Hölling ${ }^{1} \cdot$ Joachim Peinke ${ }^{1}$
}

Received: 14 May 2021 / Accepted: 10 December 2021 / Published online: 20 January 2022

(c) The Author(s) 2022

\begin{abstract}
In free-field operation, many aerodynamic systems are confronted with changing turbulent inflow conditions. Wind turbines are a prominent example. Here, the rotation of the rotor blades causes incoming wind gusts to result in a local change in the angle of incidence for the blade segments, which changes the effective angle of attack and can lead to dynamic non-linear effects like dynamic stall. Dynamic stall is known to produce a significant overshoot in the acting forces and thus an increase in loads acting on the wind turbine, leading to long-term fatigue. To gain a better understanding, it is necessary to perform wind tunnel experiments under realistic and reproducible inflow with defined conditions. In this study, a so-called 2D active grid is presented, which allows the generation of defined two-dimensional inflow conditions for wind tunnel experiments. The focus is on generating sinusoidal transversal and longitudinal gusts with high amplitudes and frequencies. Different grid configurations and sizes are tested to investigate differences in the generated flow fields. Transversal gusts imposed in this way can be used to study dynamic phenomena without having to move the object under investigation itself. Inertial effects during force measurements and a changing shadow casting due to moving airfoils in particle image velocimetry measurements are thus avoided. The additional possibility to generate defined longitudinal gusts allows to generate a broad range of reproducible inflow situations like yaw or tower shadow effects during experimental investigations.
\end{abstract}

Keywords Active Grid · Gust Generation · Flow Modulation · Dynamic Stall · Wind Tunnel Testing

\section{Introduction}

In nature, all kinds of objects are often exposed to rapidly changing inflow conditions. Consequently, the aerodynamics of these objects becomes challenging and a highly nonlinear phenomenon. Typical prominent examples of applications which suffer from dynamic inflow are helicopters or wind turbines. For helicopters, the complex inflow results mainly

Tom T. B. Wester

tom.wester@uni-oldenburg.de

1 ForWind, Institute of Physics, University of Oldenburg, Küpkersweg 70, Oldenburg, Germany 
from the rotor motion. The advancing or retreating blade causes a change in relative velocity. In addition, the blades are pitched with each rotation, resulting in a pitching and/or plunging motion. The resulting effects have therefore been studied extensively for decades (Harris and Pruyn 1968; Liiva 1969; Leishman 2006). In the case of wind turbines, especially in the root region, the dynamic effects do not result primarily from the movement of the blades themselves. Here, the turbulence impinging on the turbine and the resulting velocity fluctuations are responsible. The blade in the rotating system perceives such changes as variations in the direction of incoming flow. Such changing flow conditions lead to dynamic loads on the blades. These loads subsequently lead to structural fatigue and are also passed on to the wind turbine's drive train and power electronics. This typically leads to increased fatigue of the entire system and thus to a shortening of the service life (Spinato et al. 2009). The amplitude of such angle of incidence (AoI) changes or transversal gusts can easily exceed several degrees depending on the turbine operating point and span position (Singh et al. 2021).

In the two examples given, dynamic stall is most likely the governing aerodynamic effect. Dynamic stall was first observed almost a century ago in two-dimensional measurements of a pitching Göttingen 459 airfoil (Kramer 1932). Up to now most experiments and CFD simulations are performed using pitching airfoils (McCroskey and Fisher 1972; Mulleners and Raffel 2013; Choudhry et al. 2014; Dunne and McKeon 2015) to induce dynamic stall. For these experiments, a pitching motion of more than $5^{\circ}$ is usually used to produce a sufficiently large dynamic stall cycle. However, as mentioned above, for wind turbine rotors the dynamic stall is mainly a consequence of the changing inflow and not of the pitching blades. Although studies have shown that the effects which occur are comparable (Gharali and Johnson 2012; Rival and Tropea 2010), the situation at hand is different and becomes even more complex on smaller time scales. For this reason, experiments must account for this difference and use a varying inflow with a fixed rather than a pitching blade.

To this end, for more than half a century, there have been various experimental setups with different devices dealing with the generation of transversal gusts and changes in angle of incidence. A plot of achieved transversal gust amplitudes in different studies using closed test sections is shown in Fig. 1. Here, the maximum amplitude $\hat{\phi}$ achieved in each study is plotted against the wind velocity $u_{\infty}$. Larger amplitudes could only be achieved by using an open jet wind tunnel (Ricci and Scotti 2008; Lancelot et al. 2017; Kuzmina et al. 2006; Saddington et al. 2015). Since the fluid dynamic fundamentals are different in open jet wind tunnel, these studies are not included in Fig. 1. In addition to the studies shown here for the lower velocity range, the successful application of oscillating shafts could also be demonstrated in transonic wind tunnels (Gilman and Bennett 1966; Reed III 1981; Neumann and Mai 2013; Brion et al. 2015). Here, however, the amplitudes are reported to be below $1.5^{\circ}$. The area of transverse gusts covered by the previous studies is shown as a gray area in Fig. 1.

From Fig. 1 it becomes apparent that the amplitudes drop very steeply with increasing wind speed. This results in a large gap for the usual wind speed $\left(u_{\infty}>10 \frac{\mathrm{m}}{\mathrm{s}}\right)$ and Reynolds number ranges $\left(\operatorname{Re}>10^{5}\right)$ for aerodynamic applications, which cannot be filled with the previous setups.

In addition to the need to keep up with the latest experiments on dynamic stall, there is also a need to support theoretical considerations with experimental data. The effect of incoming transversal gusts on airfoils was already theoretically studied at the beginning of the twentieth century by Sears (1938). He described the transversal gust as a distribution of vortices along the airfoil camber. This theory was later expanded by Goldstein and Atassi 


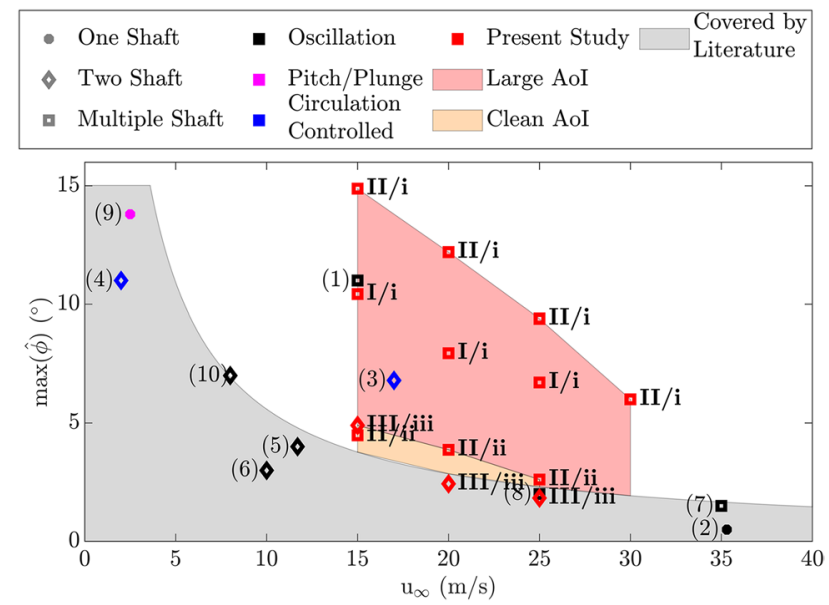

Fig. 1 Maximal achieved transversal gust amplitudes in the literature as a function of velocity based on following studies: (1) Grissom and Devenport (2004), (2) Hakkinen and Richardson Jr (1957), (3) Ham et al. (1974), (4) Jancauskas and Melbourne (1986), (5) Stapountzis (1982), (6) Wu et al. (2013), (7) Kobayakawa and Maeda (1978), (8) Tang et al. (1996), (9) Wei et al. (2019a), (10) Wood et al. (2017). The symbols indicate the number of shafts used for flow redirection [one (o), two $(\diamond)$, multiple $(\quad \square)$ ]. The approach is indicated by the color (oscillation (black), pitch/plunge (pink), circulation controlled (blue). The gray area indicates what has been shown in closed test sections with oscillating shafts so far in literature. The results of this study are plotted in red, and the newly covered area for very clean AoI modulations in orange and very large AoI variations in red

(1976) and Atassi (1984) with second order models. Proving these theories experimentally turned out to be very difficult. The use of a classical active grid (Makita and Sassa 1991; Knebel et al. 2011; Reinke 2017) for flow modulation was the first way to achieve agreement between theory and experiment (Cordes et al. 2017). Subsequent investigations of such active grids have revealed three-dimensional structures within the generated flow (Traphan et al. 2020). These three-dimensional structures are probably the reason for the appearance of unwanted aerodynamic effects and thus led to ambiguity in the experimental realization of the theories of Sears and Atassi.

In this study, a so-called 2D active grid is presented. With this grid, significantly larger inflow variations can be generated in closed wind tunnel test sections than was previously possible. The new area accessed by the device is shown in Fig. 1 for the different configurations presented later as red and orange area, respectively. The different configurations can significantly affect the quality of the flow, producing flows capable to test the theories of Sears (pure transversal gusts, no fluctuations in the longitudinal component) and Atassi (a transversal gust and additional modulation of the longitudinal component). These results can be found in Wei et al. (2019b). In addition to presenting results, the study is intended to serve as guideline for replicating such an $2 \mathrm{D}$ active grid. For this reason, dimensionless numbers are used, which can subsequently be scaled for arbitrary replicates. Furthermore, the scalability is shown in this study by a small replica of the $2 \mathrm{D}$ active grid.

As previously mentioned, the 2D active grid can induce not only transversal, but also longitudinal gusts on small time scales. In general, such velocity gusts can be generated using different approaches. In Neunaber and Braud (2020), a new type of device called "Chopper" was introduced, which generates velocity fluctuations of up to $0.4 \mathrm{~Hz}$ in the test section. In Greenblatt (2016), an overview of louver systems for modulating flow velocity 
is given, but no modulation with more than $4.2 \mathrm{~Hz}$ is mentioned. Recently Farnsworth et al. (2020) introduced the idea of using a louver system in front of the wind tunnel fan to modulate the inflow velocity with up to $1.5 \mathrm{~Hz}$. The downstream louver systems show significant advantages in the quality of the gusts produced but are also limited to this application. In the 2D active grid, the separability of the individual shaft motions allows one part to generate transversal gusts and another part to generate longitudinal gusts, allowing a combination of both with a single device.

This study is structured as follows. First the experimental setup is presented in Sect. 2. This includes the wind tunnels in Sect. 2.1, the 2D active grids in Sect. 2.2, with its different configurations, shaft shapes and sizes as well as the general measurement parameter in Sect. 2.3. In the following, different realized inflows are discussed in Sect. 3. In a first step the two-dimensionality of the generated flow field is shown in Sect. 3.1. In Sect. 3.2 possible longitudinal gusts are presented. Subsequently, Sect. 3.3 compares the achievable AoI variations between the different grid configurations, followed by a demonstration of scalability in Sect. 3.4. Finally, Sect. 4 concludes the paper.

\section{Experimental Setup}

\subsection{Wind Tunnel}

The main experiments are performed in a wind tunnel with a closed test section. A sketch of the tunnel is shown in Fig. 2. It is a closed return wind tunnel with a maximal speed of $50 \mathrm{~m} / \mathrm{s}$. The closed test section has a cross section of $(0.8 \times 1.0) \mathrm{m}^{2}(h \times w)$ and a length of $2.6 \mathrm{~m}$. The side walls, the top and bottom of the closed test section are made of acrylic glass to allow full optical access. The figure also shows the coordinate system for the following results.

The wind tunnel is mainly used for aerodynamic experiments, including force measurements and the study of aerodynamic effects developing around airfoil models. Such objects can be mounted $1.1 \mathrm{~m}$ downstream of the nozzle. The airfoils are rotated around their quarter chord point using a stepper motor. The geometric angle of attack $\alpha$ is recorded with an angular encoder positioned underneath the test section. Aerodynamic forces are measured

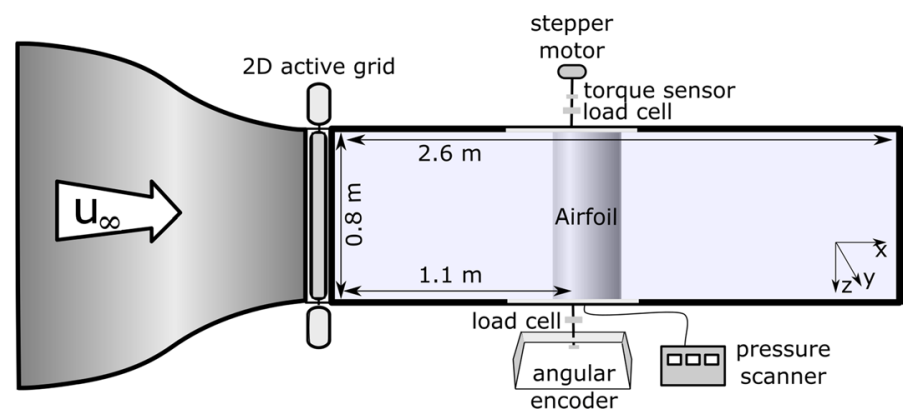

Fig. 2 Sketch of experimental setup. Inflow comes from left and passes the 2D active grid, which is mounted at the nozzle exit. The flow travels in the x-direction towards the airfoil, mounted parallel to the $\mathrm{z}$-axis in the middle of the wind tunnel 
Fig. 3 Technical drawing of the $2 \mathrm{D}$ active grid containing the stepper motors (1-9), the gauge of the shafts $g_{\text {shaft }}$, the span of the shafts $s_{\text {shaft }}$ and the chord length of the shafts $c_{\text {shaft }}$. At the right side, the definition of shaft angle $\gamma$ is shown (top view onto the shaft)

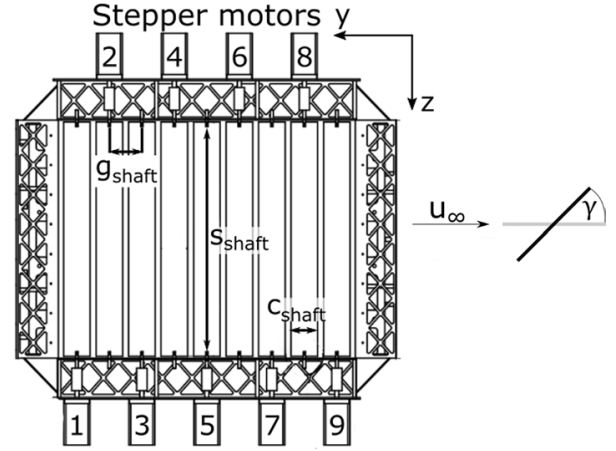

II

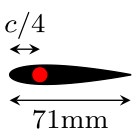

III

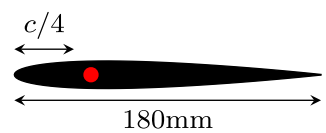

Fig. 4 Shafts for the 2D active grid. I: Flat plates with a chord length of $90 \mathrm{~mm}$. II: NACA0018 airfoils with a chord length of $71 \mathrm{~mm}$. III: NACA0009 with a chord length of $180 \mathrm{~mm}$. The red mark indicates the pivot point

using load cells with a temporal resolution of $1 \mathrm{kHz}$. These are mounted on top and bottom of the airfoil. To capture the torque, an additional sensor is placed below the stepper motor.

For scaling experiments, additional measurements are performed in a second return wind tunnel of the university. This wind tunnel is a down scaled version of the first one and has a cross section of $(0.25 \times 0.25) \mathrm{m}^{2}(h \times w)$, a length of $2 \mathrm{~m}$ and a maximal wind speed of $20 \mathrm{~m} / \mathrm{s}$. Again, the test section is made of acrylic glass for optical access.

\subsection{D Active Grid}

In this study two 2D active grids of different sizes are used, which match the outlet sizes of described wind tunnels. First, the main grid, whose results make up most of the study, is explained. Then, the smaller 2D active grid, also referred to as the replica, is specified.

The $2 \mathrm{D}$ active grid uses up to nine vertical shafts with a span of $s_{\text {shaft }}=800 \mathrm{~mm}$ and a chord length $c_{\text {shaft }}$, depending on the type. The gauge in-between shaft positions are $g_{\text {shaft }}=$ $110 \mathrm{~mm}$. A technical drawing of the grid is shown in Fig. 3. Each shaft is moved by its own stepper motor. Motion protocols, which contain the position for each time step are given to stepper motors via NI motion control boards. The angle $\gamma$ describes the orientation of the shaft with respect to the incoming flow (see Fig. 3).

In this study, three grid configurations with different shaft types (see Fig. 4) are investigated. Shaft type $\mathbf{I}$ are flat plates with a chord length $c$ of $c_{\mathrm{FP}}=90 \mathrm{~mm}$ and a thickness of $d_{\mathrm{FP}}=5 \mathrm{~mm}$. The pivot point of these shafts is at $c / 2$ to reduce inertia during the rotation. Shaft type II are 3D printed NACA0018 airfoils with a chord length of $c_{\text {NACA0018 }}=71 \mathrm{~mm}$ and a thickness of $d_{\mathrm{NACA} 0018}=12.8 \mathrm{~mm}$. For aerodynamic reasons, the pivot point of those airfoils is at $c / 4$. The shape is chosen to optimize aerodynamic properties of the shafts. Especially for large $\gamma$, aerodynamic shapes show a more benign behavior. Shaft type III are $3 \mathrm{D}$ printed NACA0009 airfoils with a chord length of $c_{\text {NACA0009 }}=180 \mathrm{~mm}$ and a thickness 
of $d_{\mathrm{NACA0009}}=16.2 \mathrm{~mm}$. Just like the NACA0018 airfoils, these are also rotated around $c / 4$.

Beside shaft geometry, also the number of shafts as well as their arrangement are varied. Figure 5 shows three configurations examined in this study. Configuration $\mathbf{i}$ shows the default setup with all nine shafts installed. This setup can be used for AoI modulations (see Sect. 3.3) or by using the outer shafts $\{1,2,8,9\}$ marked in red to modulate the blockage and therefore the longitudinal velocity (see Sect. 3.2).

In configuration ii the inner three shafts $\{4,5,6\}$ are removed. This configuration also allows the generation of transversal gusts but reduces the disturbing influence of the centerline shafts. To ensure the best possible flow quality, only shaft type II is installed in this configuration.

The third configuration iii uses only two shafts at positions $\{3,7\}$. In this configuration NACA0009 airfoils III are installed. This is the most commonly found configuration in the literature for generating AoI variations. For this reason, it is included as a reference.

To test whether the later presented results are scalable to any setup, a smaller 2D active grid replica is manufactured in addition to the previous one. This small replica fits with its dimensions of $0.25 \mathrm{~m} \times 0.25 \mathrm{~m}$ onto the nozzle of the smaller wind tunnel. Based on the square shape, this grid has only five vertical shafts instead of nine in configuration i. During experiments, these are equipped with type I shafts. Due to the smaller gauge of $g_{\text {shaft }}=$ $50 \mathrm{~mm}$ the chord is also reduced to $c_{\mathrm{FP}}=45 \mathrm{~mm}$. The shafts have a span width of $s_{\text {shaft }}=$ $245 \mathrm{~mm}$.

In general, when selecting the shafts, care must be taken to ensure that the chosen shapes are used in a Reynolds number range in which they will be functional. For this reason, for example, the NACA0018 shafts are not considered for the grid replica because the Reynolds number would be too low. In addition, the gauge of the shafts should be selected so that the shafts cannot touch each other during full rotation. Therefore, the ratio of gauge to chord should be larger than 1 . This also keeps the blockage of the wind tunnel low.

\subsection{Measurements}

In order to characterize the flow field induced by the active grids X-wire measurements are performed using probes of type 55P61 from Dantec Dynamics with a sampling rate of 20 $\mathrm{kHz}$ and a duration of $30 \mathrm{~s}$ per measurement. A Streamline $90 \mathrm{H} 02$ Flow Unit is used for the calibration of the X-wires. The error of the velocity measurement can be estimated to be $<1 \%$ (Jørgensen 2001). Measurements are taken on the centerline at the intended position of the airfoil leading edge $1 \mathrm{~m}$ downstream of the $2 \mathrm{D}$ active grid. This corresponds to

Fig. 5 Shaft setups of the 2D active grid as top view on the shafts. i shows a setup with nine shafts, ii uses six shafts and in iii only two shafts of type NACA0009 are installed

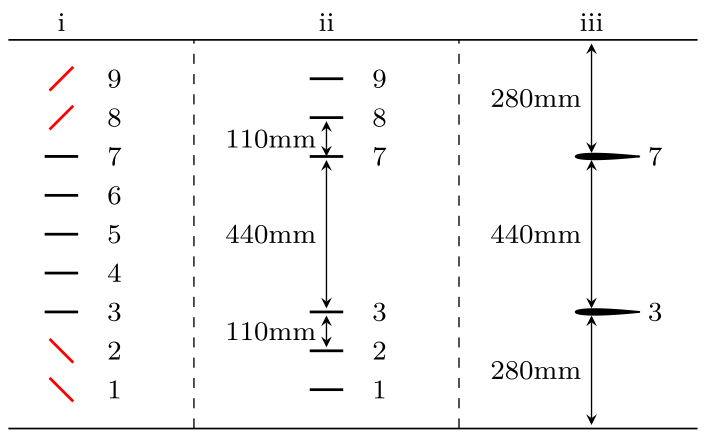


nine times the gauge of the shafts in configuration $\mathbf{i}$. To obtain comparable results for the grid replica, the distance of nine times the shaft gauge is kept. In this case, this corresponds to a distance of $0.45 \mathrm{~m}$ downstream of the grid.

In addition to hot-wire measurements, also stereoscopic PIV measurements are performed for some representative cases to investigate spatial structures behind the 2D active grid. The PIV system consists of a Litron 303HE laser, two Phantom Miro 320S cameras and a PTU high speed timing unit by LaVision. The investigated field of view covers the centerline in the middle height of the test section. During measurements a temporal resolution of $500 \mathrm{~Hz}$ is used covering an area of $0.1 \mathrm{~m}$ in $\mathrm{y}$ and $0.3 \mathrm{~m}$ in $\mathrm{x}$ direction. The total measurement duration of each measurement is limited to $5 \mathrm{~s}$ by the camera RAM.

To cover a broad range of applications of the setups, the parameters given in Table 1 are tested with the 2D active grids. The presented flows are generated using sinusoidal movements of the shafts with different frequencies $f$ in steps of $\Delta f$, amplitudes $\hat{\gamma}$ in steps of $\Delta \hat{\gamma}$ and free stream velocities of $u_{\infty}$. The last row represents the parameters for the small 2D active grid replica. Since the dimensions are smaller here, a larger parameter range could be covered.

\section{Results}

The flow modulations, which can be generated with the different configurations are shown in the following. The reduced frequency

$$
k=\frac{\pi c f}{u_{\infty}}
$$

Leishman (2006) is used to present the results. $c$ denotes the chord length of the considered shaft, $u_{\infty}$ the inflow velocity and $f$ the frequency of the shaft motion or comparably the frequency of the modulated inflow. Since $k$ considers the frequency of the gust and the free stream velocity, this quantity is ideal for comparing the results of different shaft types and velocities.

Table 1 Parameters used during the characterization of the $2 \mathrm{D}$ active grids for different setups

\begin{tabular}{llllll}
\hline Setup & $\mathrm{f}(\mathrm{Hz})$ & $\Delta \mathrm{f}(\mathrm{Hz})$ & $\hat{\gamma}\left({ }^{\circ}\right)$ & $\Delta \hat{\gamma}\left({ }^{\circ}\right)$ & $u_{\infty}(\mathrm{m} / \mathrm{s})$ \\
\hline $\mathbf{I} / \mathbf{i}$ & $1-8$ & 1 & $2.5-15$ & 2.5 & $15-25$ \\
$\mathbf{I I} / \mathbf{i}$ & $1-10$ & 1 & $2.5-20$ & 2.5 & $15-30$ \\
$\mathbf{I I} / \mathbf{i i}$ & $1-10$ & 1 & $2.5-15$ & 2.5 & $15-25$ \\
$\mathbf{I I I} /$ iii & $1-5$ & 1 & $2.5-15$ & 2.5 & $15-25$ \\
Replica I*/i & $1-15$ & 1 & $2.5-20$ & 2.5 & $5-15$ \\
\hline
\end{tabular}

I* for the replica indicates that only a configuration comparable to I was chosen

The bold characters in Table 1 correspond to the definitions of configurations and shafts. These are highlighted in bold in the entire text andfigures and are therefore also shown in this way in the table 


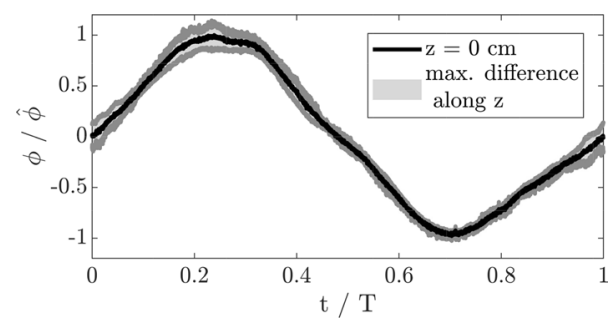

Fig. 6 Measurement of $\phi$ along the z-direction. The black line represents the $z=0 \mathrm{~mm}$ measurement. The maximum deviation between centerline and measurements at $z=(-50,-100,-150,-200,-250,-300)$ $\mathrm{mm}$ is given by the error tube in gray. The measurements are performed with setup II/i at $k=0.08$ and $\hat{\gamma}=7.5^{\circ}$ amplitude. The results are normalized by the AoI amplitude $\hat{\phi}$

Fig. 7 a Phase average PIV results of the angle of incidence modulation in $\mathbf{y}$-direction. $\mathbf{b}$ Extracted time series from a. The black line represents $y=0 \mathrm{~mm}$. The maximum deviation between centerline and the time series from $y=-55 \mathrm{~mm}$ to $y=65 \mathrm{~mm}$ ( 80 time series) is given by the error tube in gray. The measurements are performed with setup II/i at $k=0.13$ and $\hat{\gamma}=7.5^{\circ}$ amplitude. The results are normalized by the AoI amplitude $\hat{\phi}$

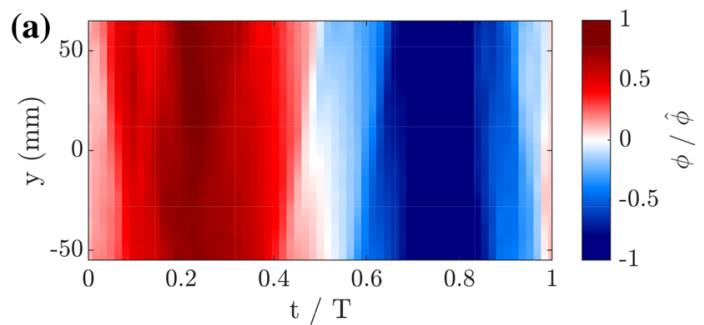

(b)

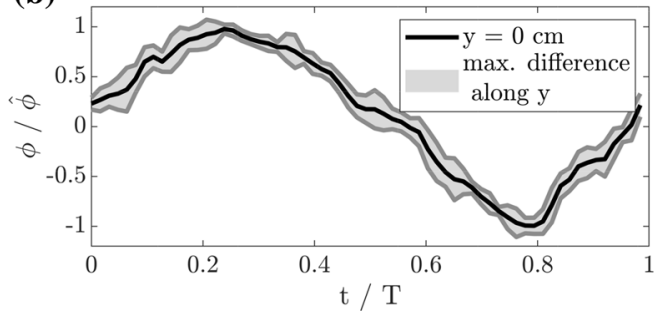

\subsection{Two-Dimensionality of the Generated Flow}

The main motivation for building a $2 \mathrm{D}$ active grid is to create a homogeneous twodimensional flow field and avoid three-dimensional flow structures along the z-direction. Previous studies have indicated such structures when a typical active grid with diamond shaped flaps is used to modulate inflow (Traphan et al. 2020). Therefore, it shall first be shown that the generated gusts are homogeneous along the transversal direction. This is achieved by means of X-wire measurements. One $\mathrm{X}$-wire is positioned on the centerline while the second $\mathrm{X}$-wire is moved in $5 \mathrm{~cm}$ steps along the z-direction. Exemplary for this comparison, the setup $\mathbf{I I} / \mathbf{i}$ is chosen and operated at a reduced frequency of $k=0.08$ with an amplitude of $\hat{\gamma}=7.5^{\circ}$. The result of the phase averaged AoI modulations is shown in Fig. 6. The centerline results (black line) show a very small deviation from the other measurement points along the z-direction, shown as gray error tubes.

To check the homogeneity in horizontal y-direction, stereoscopic PIV measurements are performed. This method is chosen to obtain a higher spatial resolution of the interaction zone of neighboring shafts and thus a better insight into the flow in general. 
Fig. 8 Overview over the longitudinal gusts generated by setup II/i. a Time series of velocity modulation induced by increasing the blockage using outer shafts. The reduced frequency is $k=0.11$ at an inflow velocity of $20 \mathrm{~m} / \mathrm{s}$. The red line shows the moving average over 500 samples of the time series. b Velocity modulation amplitudes $\hat{u}$ for a fixed blockage ratio of $18.5 \%$. The filled symbols represent the standard deviation $\hat{u}^{\prime}$ around the modulated flow. c Normalized velocity modulation amplitudes for reduced frequency of $k \approx 0.1$ at different free stream velocities using four outer shafts. The filled symbols represent the standard deviation around the modulated flow

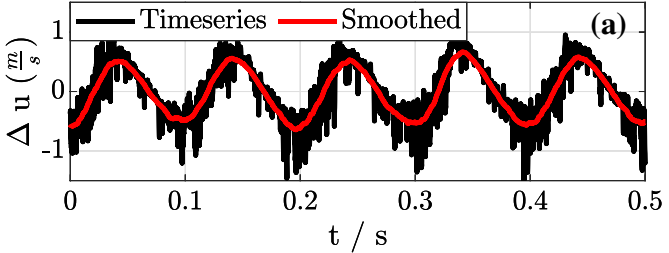

$15 \frac{m}{s} 18.5 \%$ Max. Grid Blockage

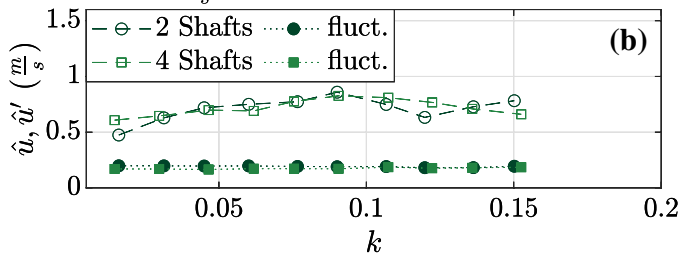

$k \approx 0.1$

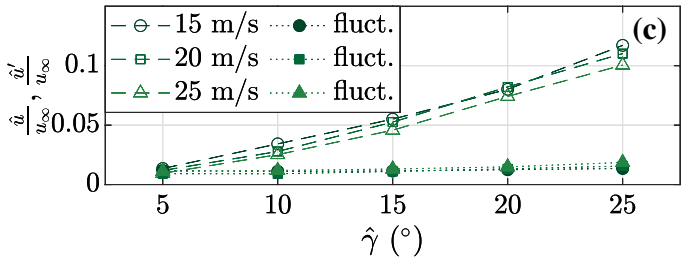

Figure 7a shows the phase averaged resulting transversal gust amplitude of a representative flow modulation. The figure shows a homogeneous modulation in the horizontal direction of the test section. In addition, Fig. 7b shows a comparison of the time series along $\mathrm{y}$-direction with respect to the centerline measurement $y=0 \mathrm{~mm}$.

Both measurements show a non-changing flow along the vertical and horizontal direction. The shape and the standard deviation of the AoI curves along $\mathrm{z}$ and $\mathrm{y}$ directions are comparable. The results also do not show a phase shift between the different measurement positions. This is a clear indication of a purely two-dimensional flow generated by the $2 \mathrm{D}$ active grid setup. The stereoscopic PIV measurements also show a small fluctuating z-component of the flow field, which is in the range of turbulence intensity and thus negligible.

\subsection{Velocity Modulation}

The following section should first investigate the generation of pure longitudinal gusts. Here velocity modulations up to $10 \mathrm{~Hz}$ can be imposed onto the flow by using the outer shafts $\{1,2,8,9\}$ with setup $\mathbf{I I} / \mathbf{i}$.

During investigation, the inner shafts remain unchanged at the open position $\gamma=0^{\circ}$, while the outer shafts perform a sinusoidal motion to periodically change the blockage. The maximum of which is given by $N \cdot c_{\text {shaft }} \cdot \sin (2 \cdot \hat{\gamma})$, where $N$ denotes the number of shafts. The motion starts at half of the desired amplitude with the shafts trailing edge pointing towards the wind tunnel walls. This is to prevent the flow from being redirected towards the centerline of the tunnel. The shafts moved between $\gamma=0^{\circ}$ and two times the amplitude $\hat{\gamma}$.

An exemplary time series resulting from such a motion is given in Fig. 8a where four outer shafts are used. The amplitude of the movement is $\hat{\gamma}=10^{\circ}$ resulting in a velocity 
modulation of $\hat{u}=0.6 \mathrm{~m} / \mathrm{s}$ at an inflow velocity of $20 \mathrm{~m} / \mathrm{s}$. The shafts move with a frequency of $f_{\text {Grid }}=10 \mathrm{~Hz}$ corresponding to a reduced frequency of $k=0.11$. The resulting longitudinal gust shows a periodic behavior close to a sinusoidal shape. Fluctuations in the valleys can be explained by the inertia of the air. This effect is well known from the literature and could be avoided e.g., by bypass ducts (see examples in Greenblatt 2016).

In the Fig. $8 \mathrm{~b}$ a comparison of the achievable velocity modulation for an induced blockage up to $18.5 \%$ is shown. Two cases with a different number of shafts in motion $(\{1,9\}$ and $\{1,2,7,9\})$ are investigated. The hollow points in the plot represent the amplitude of the velocity modulation $\hat{u}$ derived from a sinusoidal fit. The filled points represent the fluctuations $\hat{u}^{\prime}$ around the velocity modulation. The fluctuations are calculated by subtracting the smoothed curve (see Fig. 8a red line) from the time series and calculating the standard deviation of the remaining signal. The amplitude of the velocity modulation $\hat{u}$ and the fluctuations $\hat{u}^{\prime}$ seem to be independent from the reduced grid frequency $k$, which is given as horizontal axis. Furthermore, this plot emphasizes a dependency of the amplitude $\hat{u}$ just on the maximal induced blockage and not on the number of shafts which are used for generating the blockage.

Figure $8 \mathrm{c}$ shows the induced velocity modulation for three different inflow velocities over the amplitude of the shafts $\hat{\gamma}$. The resulting velocity modulations are normalized by the inflow velocity $u_{\infty}$, to compare the plots. The filled symbols represent again the fluctuations $\hat{u}^{\prime}$ around the modulation. Similar behaviors are found for all wind speeds. Hence, the velocity modulation is independent of the inflow velocity and only depending on the induced maximal blockage. This is represented by the monotonic increase of the velocity modulation when the amplitude of the shafts is also increased. Furthermore, the fluctuations $\hat{u}^{\prime}$ are constant over the entire investigated range.

In summary Fig. 8b, c show a broad range of possible velocity fluctuations and only a dependency on the blockage of the grid. Neither the high frequency of the grid, nor the inflow velocity have an impact on the resulting longitudinal gust amplitudes. A plot of the other tested parameters can be found in the supplementary material.

\subsection{Angle of Incidence Modulation}

This section will discuss the transversal gusts induced by the different setups with respect to the quality and amplitudes. Figures 9, 10,11 and 12 show an overview of flow variations generated by the configurations mentioned before. The figures show the following:

- Figure 9: Resulting phase averaged velocity components $u_{\mathrm{x}}$ (longitudinal) in blue and $u_{\mathrm{y}}$ (transversal) in orange.

- Figure 10: Resulting phase averaged angle of incidence (AoI) variation $\phi=\tan ^{-1}\left(\frac{u_{y}}{u_{x}}\right)$ in black with a sinusoidal fit in red.

- Figure 11: Resulting AoI magnitude $\hat{\phi}$ as a function of the shaft amplitude $\hat{\gamma}$.

- Figure 12: Resulting AoI magnitude $\hat{\phi}$ as a function of the reduced frequency of the grid $k$.

Figures 11 and 12 reflect a cut through the tested parameter range. The error bars of those figures indicate the deviation from a perfect sinusoidal flow. To determine these error bars, a sinusoidal fit is subtracted from the AoI time series. The standard deviation is calculated from the remaining fluctuations. 

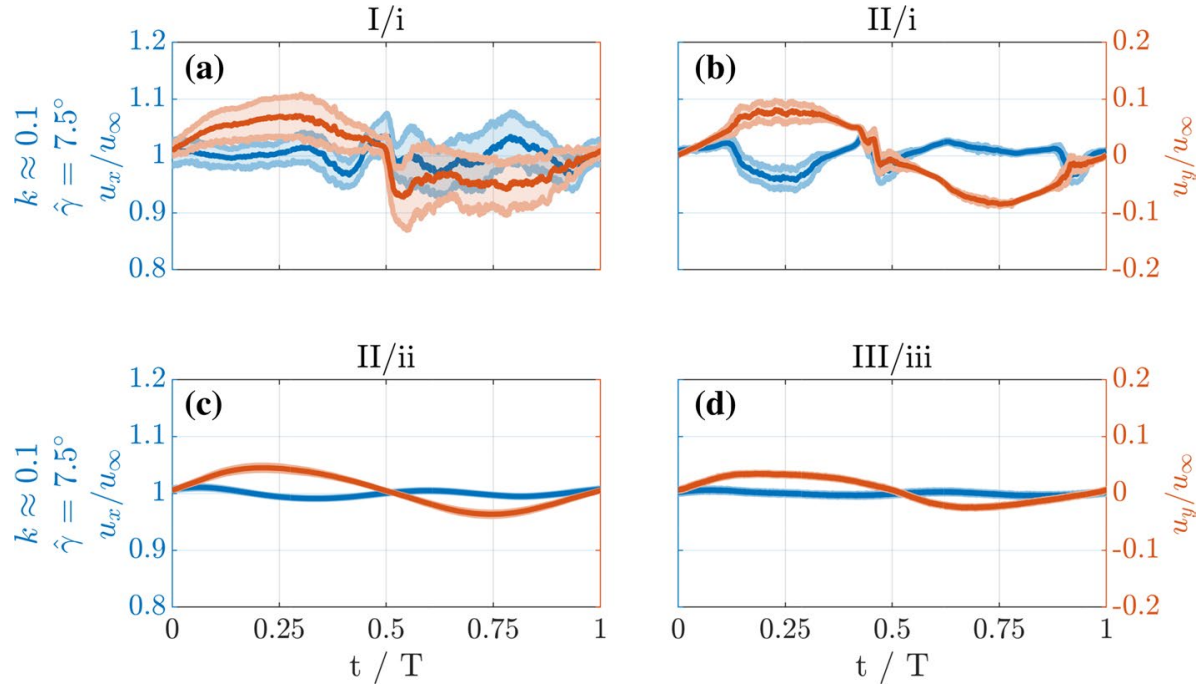

Fig. 9 Comparison of the velocity components generated by different setups. Phase averaged time signal of measured velocity components for a grid amplitude of $\hat{\gamma}=7.5^{\circ}$ at a reduced frequency of $k \approx 0.1$. The error tubes represent the standard deviation of the phase average
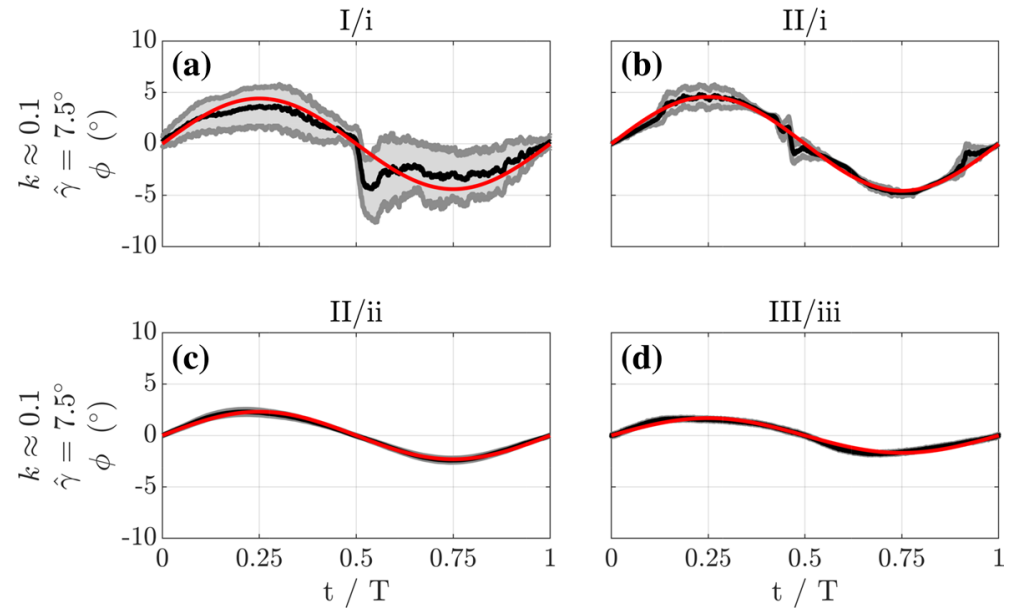

Fig. 10 Comparison of the angle of incidence generated by different setups. The figures show the phase averaged AoI. The error tubes represent the standard deviation of the phase average.

All the figures shown indicate that modulation of the flow is possible with the presented setups. Nevertheless, there are significant differences between the results. In general, these can be divided into two categories. The first category is configuration $\mathbf{i}$, where shafts are present on the centerline, and configurations ii and iii, where the centerline is vacant.

The largest difference between the two categories can be seen in Fig. 9. While configuration $\mathbf{i}$ shows fluctuations in $u_{\mathrm{x}}$ and $u_{\mathrm{y}}$, configurations $\mathbf{i i}$ and iii hardly show them. The 

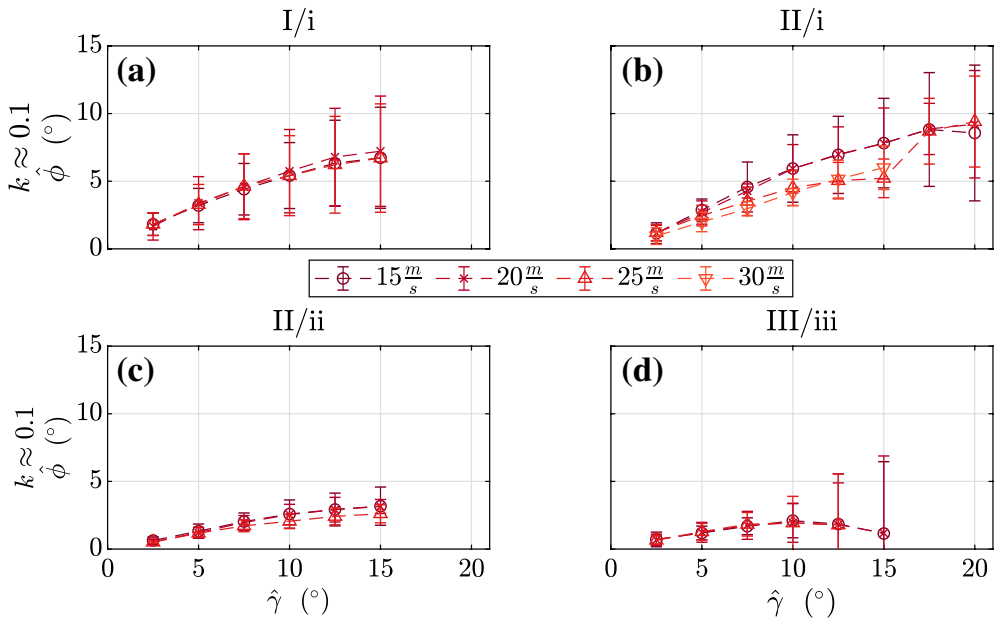

Fig. 11 Comparison of the angle of incidence amplitude depending on the shaft angle amplitude $\hat{\gamma}$. The plots show the resulting AoI amplitudes $\hat{\phi}$ for different grid amplitudes $\hat{\gamma}$ at a fixed reduced frequency of $k \approx 0.1$
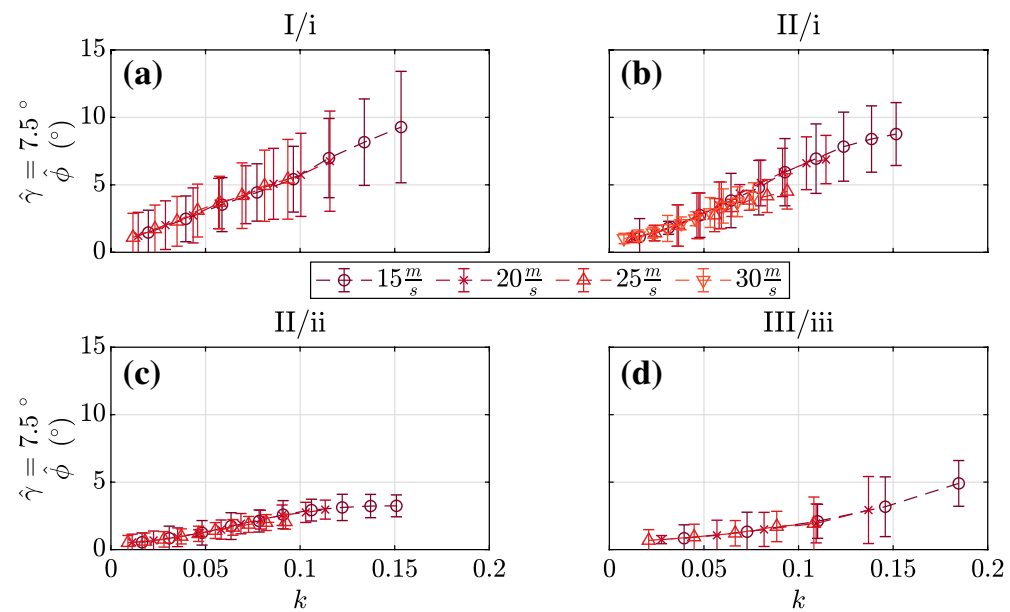

Fig. 12 Comparison of the angle of incidence amplitudes depending on the reduced frequency $k$. The figure shows the resulting AoI amplitudes $\hat{\phi}$ for different reduced frequencies of the shafts at fixed amplitude of $\hat{\gamma}=7.5^{\circ}$

explanation lies in the higher number of shafts and the resulting greater blockage during operation, as well as in the resulting wake of the centerline shaft.

However, the larger number of shafts also provides more efficient flow redirection, which means larger amplitudes of the $u_{\mathrm{y}}$ component. This also directly leads to larger transversal gust amplitudes $\hat{\phi}$, which are shown in Fig. 10. Here it is also indicated that the fluctuations of the longitudinal component have no influence on the AoI curve. The AoI is mainly determined by the transversal component. 
The by a factor of three larger amplitudes of configuration $\mathbf{i}$ compared to ii and iii are presented in Figs. 11 and 12. For all configurations tested, a monotonically increasing transversal gust amplitude $\hat{\phi}$ is observed with increasing grid amplitude $\hat{\gamma}$ (Fig. 11) as well as with increasing reduced frequency $k$ (Fig. 12).

A major difference between the two categories is the size of the error bars. While configuration $\mathbf{i}$ shows considerable fluctuations, these are negligible for the setups with fewer shafts. However, a reduction in the fluctuations seems to be achieved by changing the shafts from type I to type II. The aerodynamic shape seems to have a positive effect on the transversal gust shape and slightly increases the amplitudes while reducing $u_{\mathrm{x}}$ fluctuations.

It should be noted, however, that these shafts show a slight velocity dependence in Fig. 11b. Thus, at smaller grid amplitudes $\hat{\gamma}$, the gust amplitudes $\hat{\phi}$ with the two higher velocities initially run below the other curves. The reason for this seems to be dynamic effects on this type of shafts, which only occur above a certain Reynolds number. However, at very large grid amplitudes, these are irrelevant and the curves collapse. At this point, reference is made to the supplementary material in which the entire tested parameter range is shown. There it can also be seen that with setup II/i amplitudes of up to $\hat{\phi}=15^{\circ}$ are achievable.

In summary, the results of this section show that the arrangement of the shafts as well as their shape have a great influence on the generated transversal gusts. Depending on the application, the shape of the shafts should be chosen carefully, and it should be checked which gust amplitude is required. It is also shown that the amplitudes scale with the reduced frequency $k$ and are thus transferable between different velocities.

\subsection{Scaling of the Grid Geometry}

To show the general scalability of the results, the smaller grid replica is used (as described in Sect. 2.2). The following section, will compare the results obtained with both grids. Figure 13a presents a comparison of the AoI amplitudes $\hat{\phi}$. Considering only $\hat{\phi}$, a clear difference in the slope of the generated transversal gust amplitudes is evident. Therefore, it was decided to introduce an efficiency factor that depends on both the shaft chord length and the shaft

Fig. 13 Comparison of different grid sizes. In the legend, Replica represents the down-scaled $2 \mathrm{D}$ active grid and $2 \mathrm{D}$ AG the normal sized one. a Resulting transversal gust amplitudes $\hat{\phi}$ for two different sized grids at various velocities and a fixed grid amplitude $\hat{\gamma}=7.5^{\circ}$. b Normalized transversal gust amplitude $\hat{\phi}^{*}$ based on the results shown in $\mathbf{a}$
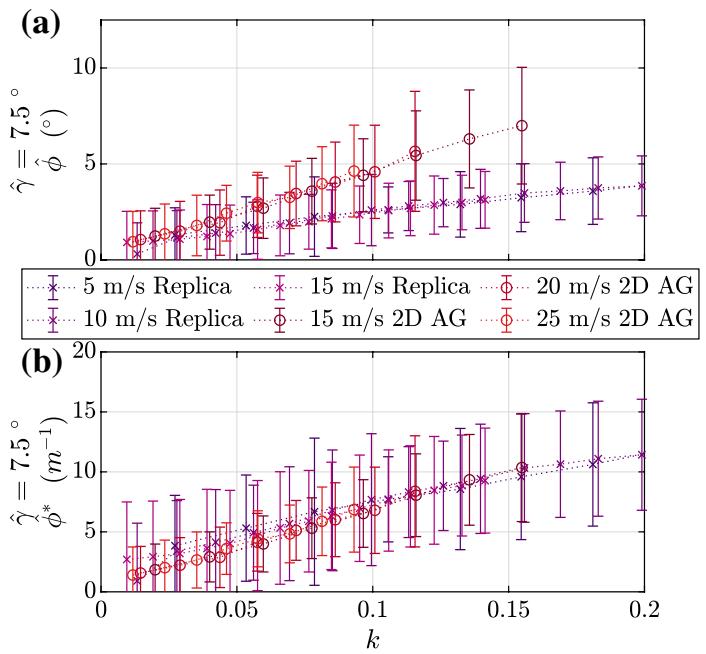
amplitude $\hat{\gamma}$. By multiplying the transversal gust amplitude with this efficiency factor, the normalized amplitude can be defined as:

$$
\hat{\phi}^{*}=\frac{1}{c \cdot \hat{\gamma}} \cdot \hat{\phi}
$$

Plotting this normalized amplitude versus $k$ for both grids results in the curve in Fig. 13b. Here all points coincide on the same curve, independent of the grid size or inflow velocity. Also the sizes of the rescaled error bars are identical for both grids.

The normalization can of course be applied to all other measurement data for all grid configurations and parameter tested. The resulting normalized AoI amplitudes $\hat{\phi}^{*}$ are shown for each configuration as a scatter plot in Fig. 14. The figure shows an alignment of all transversal gust amplitudes independent of shaft amplitude $\hat{\gamma}$ and grid size, which scales with $k$. For each configuration, a fit of the form $\hat{\phi}^{*}=a \cdot k^{b}$ can be applied on the data. This allows to estimate the resulting angle of incidence for an arbitrarily scaled configuration in advance.

Hence, the principle of the $2 \mathrm{D}$ active grid is scalable to arbitrary sizes via normalized amplitude, provided appropriate shafts are chosen for the Reynolds number range.
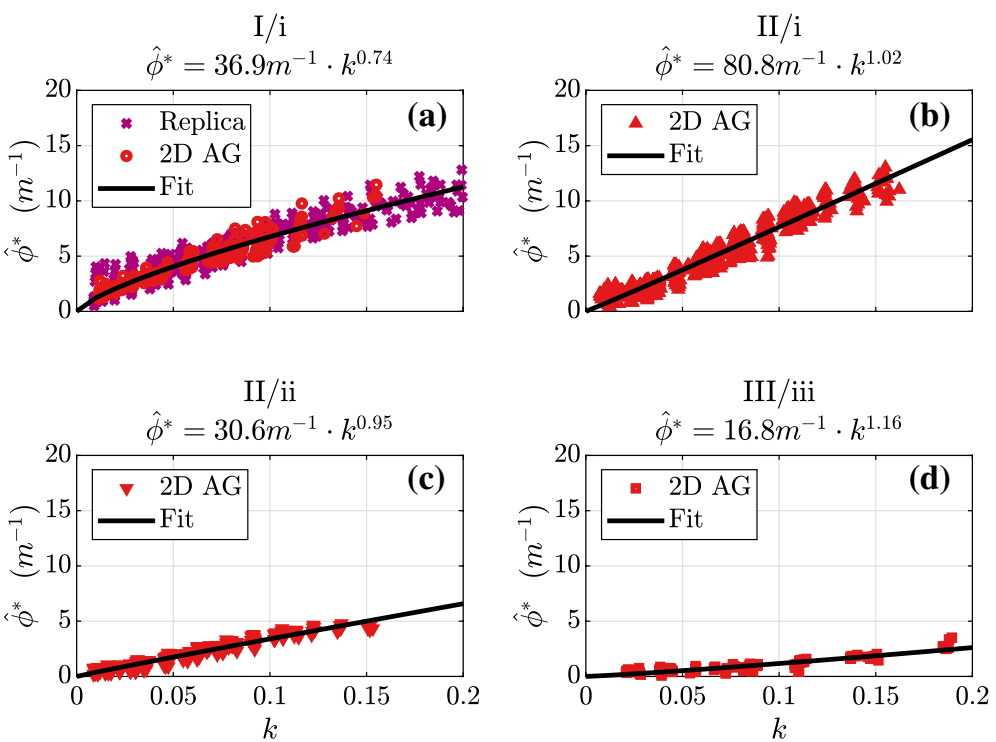

Fig. 14 The figure shows all measured and normalized AoI amplitudes $\hat{\phi}^{*}$ for all grid amplitudes $\hat{\gamma}$ and reduced frequencies $k$. The red symbols represent the measurements made with the normal sized 2D active grid, the purple crosses in plot a those measurements made with the scaled 2D active grid replica (setup equal to $\mathbf{I} / \mathbf{i}$ ). Each subplot represents one of the configurations. a shows the results of setup $\mathbf{I} / \mathbf{i}$, $\mathbf{b}$ those of II/i, c represents setup II/ii, and $\mathbf{d}$ the resulting normalized amplitudes of III/iii. The title of each subfigure contains the fit parameters, which is shown in black for each data set 


\section{Conclusion}

In this study an experimental approach to modulate a wind tunnel inflow in a highly dynamic manner is presented. The so-called 2D active grid enables the generation of purely two-dimensional inflows with desired gust amplitudes and frequencies. This holds true for the longitudinal as well as the transversal flow direction.

For the longitudinal gusts it is shown that only the blockage induced by the shafts is decisive for the gust amplitude $\hat{u}$. The variations are therefore only a function of the shaft angle $\hat{\gamma}$ and the chord length $c_{\text {shaft }}$.

For transversal gusts, six general conclusions can be drawn from the results obtained.

I. The arrangement of the shafts is crucial for the achievable transversal gust amplitude. For large amplitudes, the shafts must be distributed over the entire width of the wind tunnel. Initial results and experience indicate that the gauge to chord $\left(g_{\text {shaft }} / c_{\text {shaft }}\right)$ ratio should be about 1.1-1.5.

II. The more shafts used, the larger the variations in blockage, resulting in variations in the longitudinal velocity component.

III. The flow quality can be improved in general by using aerodynamically shaped shafts. However, attention must be paid to the Reynolds number range to avoid undesired effects.

IV. Fluctuations in the longitudinal component can be significantly reduced and a perfect transversal gust can be generated if shafts are not installed on the centerline of the tunnel. However, this comes at the expense of the achievable amplitudes.

V. The amplitudes of all setups scale with the reduced frequency $k$. Thus, the results can be scaled independently of the velocity.

VI. By using the normalized amplitude $\hat{\phi}^{*}$, the results can be scaled to arbitrary grid sizes.

With the 2D active grid, the results presented allow to keep up with the state of the art in dynamic stall experiments without relying further on pitching airfoils. Thus, it is possible to get one step closer to imitate the actual conditions at wind turbines in a wind tunnel. In addition, many experimental conditions are simplified, such as force measurements where the inertia of the pitched airfoil no longer needs to be subtracted, or additional mass effects resulting from blade motion. PIV measurements on the airfoil itself are also easier to perform, since the light sheet only needs to be aligned with a fixed object, so that the chord area detected is always the same and the shadow casts always remain in one place during the measurement.

The shown possibilities to produce various inflow conditions with a single device can further be used to generate more complex flow situations in a wind tunnel. This includes for instance yaw misalignment or tower shadow effects by using a combination of transversal and longitudinal gusts in a tailored manner.

The 2D active grid can also be used for other research areas where rapid inflow changes occur. Examples are micro air vehicles (MAV) as well as experiments on flying creatures, on whose wings dynamic effects typically play an important role. This emphasizes the broad applicability of the device presented here.

Supplementary Information The online version contains supplementary material available at https://doi. org/10.1007/s10494-021-00312-8. 
Acknowledgements The present investigations were performed within the "Wind Turbine Load Control under Realistic Turbulent In-Flow Conditions" (PE 478/15-2 \& HO 50272-2) project. The authors gratefully acknowledge the German Research Foundation (DFG) for funding the studies. The authors also want to thank Lars Kröger and Piyush Singh for their support during the measurement campaigns. The authors would also like to thank Cameron Tropea for fruitful discussions during the design phase of the 2D active grid.

Author Contributions All authors contributed to the study conception and design. Material preparation, data collection and analysis were performed by TTBW, JK and LN. AH designed the active 2D grid and was instrumental in its construction. GG, MH and JP have contributed significantly to the development of the measurement results through discussions. The first draft of the manuscript was written by TTBW and all authors commented on previous versions of the manuscript. All authors read and approved the final manuscript.

Funding Open Access funding enabled and organized by Projekt DEAL. The investigations shown in the manuscript were funded by the DFG within the "Wind Turbine Load Control under Realistic Turbulent InFlow Conditions" (PE 478/15-2 \& HO 50272-2) project.

Data Availability The data will not be deposited. All necessary information can be extracted from the shown figures.

\section{Declarations}

Conflict of interest The authors declare that they have no conflict of interest.

Open Access This article is licensed under a Creative Commons Attribution 4.0 International License, which permits use, sharing, adaptation, distribution and reproduction in any medium or format, as long as you give appropriate credit to the original author(s) and the source, provide a link to the Creative Commons licence, and indicate if changes were made. The images or other third party material in this article are included in the article's Creative Commons licence, unless indicated otherwise in a credit line to the material. If material is not included in the article's Creative Commons licence and your intended use is not permitted by statutory regulation or exceeds the permitted use, you will need to obtain permission directly from the copyright holder. To view a copy of this licence, visit http://creativecommons.org/licenses/by/4.0/.

\section{References}

Atassi, H.: The sears problem for a lifting airfoil revisited-new results. J. Fluid Mech. 141, 109-122 (1984)

Brion, V., Lepage, A., Amosse, Y., Soulevant, D., Senecat, P., Abart, J.C., Paillart, P.: Generation of vertical gusts in a transonic wind tunnel. Exp. Fluids (2015). https://doi.org/10.1007/s00348-015-2016-5

Choudhry, A., Leknys, R., Arjomandi, M., Kelso, R.: An insight into the dynamic stall lift characteristics. Exp. Thermal Fluid Sci. 58, 188-208 (2014). https://doi.org/10.1016/j.expthermflusci.2014.07.006

Cordes, U., Kampers, G., Meißner, T., Tropea, C., Peinke, J., Hölling, M.: Note on the limitations of the theodorsen and sears functions. J. Fluid Mech. 811 (2017)

Dunne, R., McKeon, B.J.: Dynamic stall on a pitching and surging airfoil. Exp. Fluids 56(8), 1-15 (2015). https://doi.org/10.1007/s00348-015-2028-1

Farnsworth, J., Sinner, D., Gloutak, D., Droste, L., Bateman, D.: Design and qualification of an unsteady low-speed wind tunnel with an upstream louver system. Exp. Fluids (2020). https://doi.org/10.1007/ s00348-020-03018-1

Gharali, K., Johnson, D.A.: Numerical modeling of an s809 airfoil under dynamic stall, erosion and high reduced frequencies. Appl. Energy 93, 45-52 (2012)

Gilman, J., Bennett, R.M.: A wind-tunnel technique for measuring frequency-response functions for gust load analyses. J. Aircr. 3(6), 535-540 (1966). https://doi.org/10.2514/3.43773

Goldstein, M.E., Atassi, H.: A complete second-order theory for the unsteady flow about an airfoil due to a periodic gust. J. Fluid Mech. 74(4), 741-765 (1976)

Greenblatt, D.: Unsteady low-speed wind tunnels. AIAA J. 1817-1830 (2016) 
Grissom, D., Devenport, W.: Development and testing of a deterministic disturbance generator. In: 10th AIAA/CEAS Aeroacoustics Conference, p. 2956 (2004). https://doi.org/10.2514/6.2004-2956

Hakkinen, R.J., Richardson, A., Jr.: Theoretical and experimental investigation of random gust loads part i: Aerodynamic transfer function of a simple wing configuration in incompressible flow (1957). https:// doi.org/10.2514/6.2004-2111

Ham, N.D., Bauer, P.H., Lawrence, T.L.: Wind tunnel generation of sinusoidal lateral and longitudinal gusts by circulation control of twin parallel airfoils. In: Massachusetts Institute of Technology, Aeroelastic and Structures Research. (1974). https://doi.org/10.2514/6.2004-3042

Harris, F.D., Pruyn, R.R.: Blade stall-half fact, half fiction. J. Am. Helicopter Soc. 13(2), $27-48$ (1968)

Jancauskas, E., Melbourne, W.: The aerodynamic admittance of two-dimensional rectangular section cylinders in smooth flow. J. Wind Eng. Ind. Aerodyn. 23, 395-408 (1986). https://doi.org/10.1016/01676105(86)90057-7

Jørgensen, F.E.: How to measure turbulence with hot-wire anemometers: a practical guide. Dantec Dyn. (2001). https://doi.org/10.1007/978-90-481-3606-3-70

Knebel, P., Kittel, A., Peinke, J.: Atmospheric wind field conditions generated by active grids. Exp. Fluids (2011). https://doi.org/10.1007/s00348-011-1056-8

Kobayakawa, M., Maeda, H.: Gust response of a wing near the ground through the lifting surface theory. J. Aircr. 15(8), 520-525 (1978). https://doi.org/10.2514/3.58400

Kramer, V.M.: Die Zunahme des Maximalauftriebes von Tragflügeln bei plötzlicher Anstellwinkelvergrösserung (boeneffekt). Z Flugtech Motorluftschiff 23, 185-189 (1932)

Kuzmina, S., Ishmuratov, F., Zichenkov, M., Chedrik, V.: Analytical-experimental study on using different control surfaces to alleviate dynamic loads. In: 47th AIAA/ASME/ASCE/AHS/ASC Structures, Structural Dynamics, and Materials Conference 14th AIAA/ASME/AHS Adaptive Structures Conference 7th, p. 2164 (2006). https://doi.org/10.2514/6.2006-2164

Lancelot, P.M., Sodja, J., Werter, N.P., De Breuker, R.: Design and testing of a low subsonic wind tunnel gust generator. Adv. Aircr. Spacecr. Sci. 4(2), 125 (2017)

Leishman, G.J.: Principles of Helicopter Aerodynamics with CD Extra. Cambridge University Press, Cambridge (2006)

Liiva, J.: Unsteady aerodynamic and stall effects on helicopter rotor blade airfoil sections. J. Aircr. 6(1), 46-51 (1969)

Makita, H., Sassa, K.: Active turbulence generation in a laboratory wind tunnel. In: Advances in Turbulence 3, pp. 497-505. Springer (1991). https://doi.org/10.1007/978-3-642-84399-0-54

McCroskey, W., Fisher, R.: Dynamic stall of airfoils and helicopter rotors. AGARD Paper 595 (1972)

Mulleners, K., Raffel, M.: Dynamic stall development. Exp. Fluids 54(2), 1469 (2013). https://doi.org/10. 1007/s00348-013-1469-7

Neumann, J., Mai, H.: Gust response: simulation of an aeroelastic experiment by a fluid-structure interaction method. J. Fluids Struct. 38, 290-302 (2013)

Neunaber, I., Braud, C.: Aerodynamic behavior of an airfoil under extreme wind conditions. J. Phys. Conf. Ser. 1618, 032035 (2020)

Reed, W.H., III.: Aeroelasticity matters: some reflections on two decades of testing in the NASA Langley transonic dynamics tunnel (1981). https://doi.org/10.2514/6.1986-1888

Reinke, N.: Application, generation and analysis of turbulent flows. Ph.D. Thesis, Universität Oldenburg (2017)

Ricci, S., Scotti, A.: Wind tunnel testing of an active controlled wing under gust excitation. In: 49th AIAA/ ASME/ASCE/AHS/ASC Structures, Structural Dynamics, and Materials Conference, 16th AIAA/ ASME/AHS Adaptive Structures Conference, 10th AIAA Non-Deterministic Approaches Conference, 9th AIAA Gossamer Spacecraft Forum, 4th AIAA Multidisciplinary Design Optimization Specialists Conference, p. 1727 (2008). https://doi.org/10.2514/6.2008-1727

Rival, D., Tropea, C.: Characteristics of pitching and plunging airfoils under dynamic-stall conditions. J. Aircr. 47(1), 80-86 (2010)

Saddington, A., Finnis, M., Knowles, K.: The characterisation of a gust generator for aerodynamic testing. Proc. Inst. Mech. Eng. Part G J. Aerosp. Eng. 229(7), 1214-1225 (2015). https://doi.org/10.1177/ 0954407015596274

Sears, W.R.: A systematic presentation of the theory of thin airfoils in non-uniform motion. Ph.D. Thesis, California Institute of Technology (1938)

Singh, P., Neuhaus, L., Huxdorf, O., Riemenschneider, J., Wild, J., Peinke, J., Hölling, M.: Experimental investigation of an active slat for airfoil load alleviation. J. Renew. Sustain. Energy 13(4), 043304 (2021). https://doi.org/10.1063/5.0045846

Spinato, F., Tavner, P.J., Van Bussel, G., Koutoulakos, E.: Reliability of wind turbine subassemblies. IET Renew. Power Gener. 3(4), 387-401 (2009) 
Stapountzis, H.: An oscillating rig for the generation of sinusoidal flows. J. Phys. E: Sci. Instrum. 15(11), 1173-1176 (1982). https://doi.org/10.1088/0022-3735/15/11/012

Tang, D., Cizmas, P.G., Dowell, E.: Experiments and analysis for a gust generator in a wind tunnel. J. Aircr. 33(1), 139-148 (1996)

Traphan, D., Wester, T.T.B., Melius, M., Peinke, J., Gülker, G., Cal, R.B.: Dynamic stall of an airfoil under tailored three-dimensional inflow conditions (2020). arXiv:200307840

Wei, N.J., Kissing, J., Tropea, C.: Generation of periodic gusts with a pitching and plunging airfoil. Exp. Fluids 60(11), 166 (2019a). https://doi.org/10.1007/s00348-019-2815-1

Wei, N.J., Kissing, J., Wester, T.T.B., Wegt, S., Schiffmann, K., Jakirlic, S., Hölling, M., Peinke, J., Tropea, C.: Insights into the periodic gust response of airfoils. J. Fluid Mech. 876, 237-263 (2019b). https:// doi.org/10.1017/jfm.2019.537

Wood, K.T., Cheung, R.C., Richardson, T.S., Cooper, J.E., Darbyshire, O., Warsop, C.: A new gust generator for a low speed wind tunnel: Design and commissioning. In: 55th AIAA Aerospace Sciences Meeting, p. 0502 (2017) https://doi.org/10.2514/6.2017-0502

Wu, Z., Chen, L., Yang, C.: Study on gust alleviation control and wind tunnel test. Sci. China Technol. Sci. 56(3), 762-771 (2013). https://doi.org/10.1007/s11431-013-5131-7 